\title{
Holistic Cyber-Physical Management for Dependable Wireless Control Systems
}

\author{
YEHAN MA, DOLVARA GUNATILAKA, and BO LI, Washington University in St. Louis, USA \\ HUMBERTO GONZALEZ, BlackThorn Therapeutics, USA \\ CHENYANG LU, Washington University in St. Louis, USA
}

\begin{abstract}
Wireless sensor-actuator networks (WSANs) are gaining momentum in industrial process automation as a communication infrastructure for lowering deployment and maintenance costs. In traditional wireless control systems, the plant controller and the network manager operate in isolation, which ignores the significant influence of network reliability on plant control performance. To enhance the dependability of industrial wireless control, we propose a holistic cyber-physical management framework that employs runtime coordination between the plant control and network management. Our design includes a holistic controller that generates actuation signals to physical plants and reconfigures the WSAN to maintain the desired control performance while saving wireless resources. As a concrete example of holistic control, we design a holistic manager that dynamically reconfigures the number of transmissions in the WSAN based on online observations of physical and cyber variables. We have implemented the holistic management framework in the wireless cyber-physicalsimulator (WCPS). A systematic case study is presented based on two five-state plants and a load positioning system using a 16-node WSAN. Simulation results show that the holistic management design has significantly enhanced the dependability of the system against both wireless interferences and physical disturbances, while effectively reducing the number of wireless transmissions.
\end{abstract}

CCS Concepts: • Networks $\rightarrow$ Sensor networks; • Computer systems organization $\rightarrow$ Sensor networks;

Additional Key Words and Phrases: Cyber-physical system, wireless control, wireless sensor-actuator network, model predictive control

\section{ACM Reference format:}

Yehan Ma, Dolvara Gunatilaka, Bo Li, Humberto Gonzalez, and Chenyang Lu. 2018. Holistic Cyber-Physical Management for Dependable Wireless Control Systems. ACM Trans. Cyber-Phys. Syst. 3, 1, Article 3 (August 2018), 25 pages.

https://doi.org/10.1145/3185510

\section{INTRODUCTION}

With the adoption of industrial wireless standards such as WirelessHART [17] and ISA100 [43], wireless sensor-actuator networks (WSANs) are being deployed in process industries worldwide.

This work was sponsored by NSF through grants 1320921 (NeTS) and 1646579 (CPS), and by the Fullgraf Foundation.

Authors' addresses: Y. Ma, D. Gunatilaka, and B. Li, Washington University in St. Louis, Computer Science and Engineering, St. Louis, MO, 63130; emails: \{yehan.ma, dgunatilaka, b.li\}@wustl.edu; H. Gonzalez, BlackThorn Therapeutics, San Francisco, CA, 94103; email: hgonzale@gmail.com; C. Lu, Washington University in St. Louis, Computer Science and Engineering, St. Louis, MO, 63130; email: Lu@wustl.edu.

Permission to make digital or hard copies of all or part of this work for personal or classroom use is granted without fee provided that copies are not made or distributed for profit or commercial advantage and that copies bear this notice and the full citation on the first page. Copyrights for components of this work owned by others than ACM must be honored. Abstracting with credit is permitted. To copy otherwise, or republish, to post on servers or to redistribute to lists, requires prior specific permission and/or a fee. Request permissions from permissions@acm.org.

(C) 2018 Association for Computing Machinery.

2378-962X/2018/08-ART3 \$15.00

https://doi.org/10.1145/3185510

ACM Transactions on Cyber-Physical Systems, Vol. 3, No. 1, Article 3. Publication date: August 2018. 

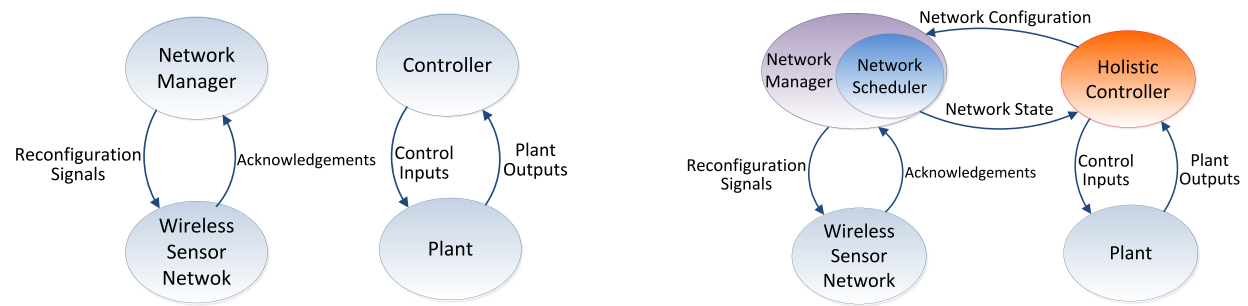

Fig. 1. Traditional design (left) vs. holistic co-design (right) of wireless process control systems.

However, existing WSANs in process industries are usually used for monitoring applications. There remain significant challenges in supporting feedback control systems over WSANs due to concerns about the dependability of wireless control systems (WCSs). A WCS employs a WSAN as the communication infrastructure for one or more feedback control loops, where the sensors, controllers, and actuators communicate over the WSAN. Despite considerable efforts to enhance the reliability of industrial WSANs, data loss is inevitable in open and harsh operating environments, which may lead to severe degradation of control performance or even system instabilities. A dependable WCS therefore must maintain system stability and acceptable control performance despite both physical disturbance and wireless interference. A WCS is particularly vulnerable when significant data loss coincides with the plant experiencing a poor physical state. The challenge of utilizing the WSANs for feedback control prevents process industries from exploiting the full potential of wireless technologies, forcing plants to maintain extensive wired infrastructure despite the existence of the WSAN. Therefore, economy, safety, and efficiency all motivate the development of WCS that are dependable under challenging cyber and physical conditions.

Although control system performance is heavily influenced by WSAN reliability, traditionally the physical plant and the network are controlled separately at runtime. The physical plant is run by a controller, designed based on certain assumptions about the communication network. However, the unpredictable wireless conditions of a WSAN mean that the wireless network design goals cannot be guaranteed, leading to unsafe physical plant operations. Moreover, the network is managed to reduce data loss in a best effort fashion, without any knowledge of the current requirements of the control system. Notably, the required level of network reliability depends on the physical states of the plant. When the physical plant is in an unsafe state, it requires highly reliable communication. Conversely, when the physical plant is in a safe steady state, it is more tolerant to data loss, which may in turn allow the network to conserve network resources.

Building on this insight, we propose a holistic cyber-physical management framework where the wireless network and the physical controller operate in a closed-loop fashion. In this holistic management approach, the controller is endowed with the capability of modifying network configurations in addition to the physical plant itself, as shown in Figure 1. The controller that provides control commands to both the physical plant and the WSAN is regarded as the holistic controller. By coordinating the control and network management at runtime, the holistic management approach can enhance the dependability of the WCS in the face of both physical disturbance and wireless interference. It can also improve the network efficiency when the plant state allows data loss.

The contributions of this work are fourfold:

(1) We propose the holistic management framework to enhance the dependability of WCS through coordinated control and network management. 
(2) We design a holistic controller based on a model predictive control scheme that simultaneously controls the physical plant while adjusting network configurations based on the state of the physical plant.

(3) We present an example holistic management design that employs a network adaptation algorithm that dynamically adjusts the number of retransmissions used to send certain actuation commands based on the physical plant conditions.

(4) We demonstrate through simulations that our holistic management scheme can enhance system dependability under both sensor disturbance and wireless interference, while avoiding allocating retransmissions not needed for the purpose of control.

The rest of the article is organized as follows: Section 2 describes our results in the context of related work. Section 3 presents the system architecture of our wireless networked control system. Section 4 presents the design of our holistic controller and its adaptation algorithm. Section 5 presents an implementable solution for a runtime WSAN reconfiguration, and Section 6 presents our experimental results.

\section{RELATED WORK}

Networked control systems are some of the best examples of cyber-physical systems, and therefore have been extensively studied in the past decade. Thus, extensions of the traditional Kalman filter have been proposed for state estimation based on intermittent observations [18, 38, 44], enabling the use of lossy networks and delayed information in feedback control applications. New sampling methods, such as event-based and self-triggered control, have been developed to reduce communication traffic over wireless networks [2,40]. These results have addressed important problems related to cyber-physical co-design, and have validated the intuition that more network resources are needed when the physical states are away from a stable equilibrium point, while few network resources are needed when the physical states are close to a stable steady-state. However, scheduling the number of packets that a network must transmit to control a system is only one side of the problem, since networks in general, and wireless networks in particular, can choose among many configurations (e.g., number of retransmissions, scheduling, and routing) to deliver those packets $[6,7,16,26,30,31,33]$. In other words, instead of abstracting the network as a transparent mechanism to transfer control information when needed, networked controllers should bidirectionally interact with the network manager as the dynamics of the physical system evolve. That is, the control system and the network should be managed in a dynamic and holistic manner.

Dynamic stability has been demonstrated under several network configurations and communication conditions, including studies of delayed packet delivery and non-independent packet losses [9, 25, 29, 32]. However, stability guarantees for networked control systems typically come in the form of minimal requirements that a network must guarantee, such as bounds in data loss or in latency, thus disregarding cooperative approaches to adapt network conditions to physical plant performance, and vice versa. Even network protocols designed with control systems in mind, such as WirelessHART [17], can guarantee only a specific level of reliability and performance under certain assumptions, thus potentially violating the sufficient conditions established in those theoretical results, especially when there are cyber or physical disturbances, or even malicious attacks. Indeed, in the case of industrial wireless environments, these disturbances take the form of cross-protocol interference, physical obstacles, power failures, extreme weather, and sensor failures, among many others.

The impacts of network retransmissions as well as the sampling rate on control performance have been studied in wireless control design. Previous works [3, 11, 13, 34] explored the tradeoffs between the sampling period (latency), reliability, network energy consumption, and the overall 
system performance. While those works focused on either control design or static network and control co-design, our holistic management framework can dynamically change network configurations based on the states of the physical systems at runtime. As an example of holistic control, we employ retransmissions as the mechanism to manipulate network reliability in response to physical system states. In the future, we plan to incorporate other adaptation mechanisms, such as sampling rates, in holistic control.

From a networking perspective, several groups have worked on tailoring wireless network protocols for control systems applications. For example, a cooperative MAC method was proposed to maintain control performance under unbounded delay, burst of packet loss, and ambient wireless traffic [42]. Bernardini and Bemporad have proposed a communication strategy between sensors and the controller that minimizes the data exchange over the wireless channel [4]. Also, several groups have considered specific scheduling and control schemes for closed-loop systems with stability guarantees $[3,12,14,20,24,35]$. Our effort is complementary to those works, in that we aim to develop a holistic cyber-physical management framework for WCSs instead of developing new network protocols.

In our previous studies $[22,23]$, we have experimentally investigated the effect of harsh network conditions on control loops. We have also developed specific routing and scheduling protocols to mitigate their effect. In this article, we build upon our previous results, developing a holistic management scheme that, instead of asking the network for a fixed minimum set of performance conditions, updates both the control algorithm and network configurations, resulting in robust and safe physical execution and efficient network information flow. As shown in Section 6, our holistic management scheme enhances the dependability of the closed-loop system, even in the presence of high wireless background noise and sensor malfunctions.

\section{A WIRELESS CONTROL SYSTEM}

Since the working conditions of industrial plants are typically too harsh for controllers to operate, and since one industrial PC is supposed to control multiple loops, controllers are always located far away from the plants in wireless industrial process control deployments. Therefore, standard industrial WCSs use multi-hop networks, such as ISA [43], WirelessHART [17], and ZigBee [47], to deliver information from a collection of sensors to a remote controller and then back from the controller to the actuators in the plant. In this article, we adopt and enhance a state-of-the-art cyber-physical architecture for industrial WCSs. On the physical side, we adopt resilient control designs that integrate a model predictive controller, a state observer, and an actuation signal buffer. On the cyber side, we employ the WirelessHART standard to enhance network reliability. Below, we explain in detail how each of the components in our architecture interacts to close the control feedback loop.

\subsection{Physical Plant and Controller}

Figure 2 shows the closed-loop system architecture that we consider in this article, which closely follows to the architecture we considered in Reference [22]. We assume that the plant is a linear time-invariant system, governed by the following difference equation:

$$
x_{t+1}=A x_{t}+B u_{t}, \quad y_{t}=C x_{t}, \quad t \in \mathbb{N},
$$

where $t$ is the time index, $x_{t} \in \mathbb{R}^{n}$ is the state vector, $u_{t} \in \mathbb{R}^{m}$ is the input vector, $y_{t} \in \mathbb{R}^{p}$ is the output vector, $A \in \mathbb{R}^{n \times n}, B \in \mathbb{R}^{n \times m}$, and $C \in \mathbb{R}^{p \times n}$. We assume that the pair $(A, B)$ is controllable and that the pair $(A, C)$ is observable.

Sensor measurements $y_{t}$ are transmitted to a remote controller via the wireless network. At time $t$, the wireless network delivers a vector $\hat{y}_{t}$, which is either equal to $y_{t-1}$ or is a no-data signal if 


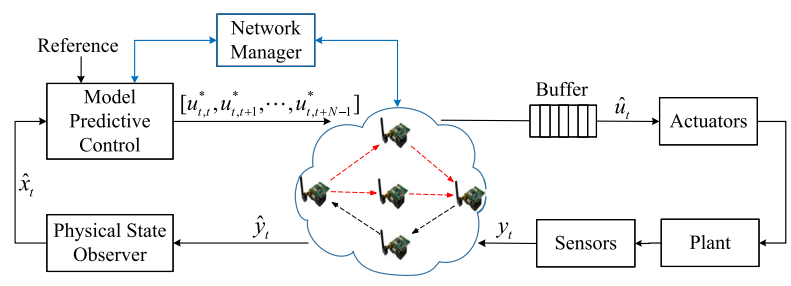

Fig. 2. System architecture.

the packet is unable to be delivered within the time sampling deadline. The vector $\hat{y}_{t}$ is delivered to an intermittent observation Kalman filter [38], which produces an estimated state vector $\hat{x}_{t}$ regardless of whether the wireless network was capable of delivering the sensing information.

At the core of the remote controller is a model predictive control (MPC) scheme that at each time $t$ solves the following optimal control problem:

$$
\begin{aligned}
V\left(\hat{x}_{t}\right)=\min _{\left\{u_{j}\right\}_{j=t}^{t+N-1}} & \sum_{j=t}^{t+N-1}\left(x_{j}^{T} Q x_{j}+u_{j}^{T} R u_{j}\right)+x_{t+N}^{T} S x_{t+N}, \\
\text { subject to } \quad & x_{t}=\hat{x}_{t}, \\
& x_{j+1}=A x_{j}+B u_{j}, \\
& x_{j} \in \mathcal{X}, u_{j} \in \mathcal{U}, j \in\{t, \ldots, t+N-1\}, \\
& x_{t+N} \in \mathcal{X}_{f},
\end{aligned}
$$

where $N \geq 0$ is the time horizon, $Q, S \in \mathbb{R}^{n \times n}$ are positive semi-definite, $R \in \mathbb{R}^{m \times m}$ is positive definite, $\mathcal{U} \subset \mathbb{R}^{m}$ is the input constraint set, and $\mathcal{X}, \mathcal{X}_{f} \subset \mathbb{R}^{n}$ are the state safety and final constraint sets, respectively. We denote by $\left\{u_{t, j}^{*}\right\}_{j=t}^{t+N-1}$ the optimal input signal at time $t$, which is the minimizer associated with value $V\left(\hat{x}_{t}\right)$ in Equation (2). Note that the controller in Equation (2) can also be used to control the system in Equation (1) around any state reference $\bar{x} \in \mathcal{X}$, satisfying

$$
(A-I) \bar{x}+B \bar{u}=0 \text {, for some } \bar{u} \in \mathcal{U} .
$$

We assume that $\bar{x} \in \mathcal{X}$ and $\bar{u} \in \mathcal{U}$.

The MPC scheme in Equation (2) sends the resulting sequence of optimal inputs, $\left\{u_{t, j}^{*}\right\}_{j=t}^{t+N-1}$, over the wireless network. Whenever the sequence successfully traverses the wireless network, it overwrites the old sequence stored in a buffer that periodically feeds the plant actuators. Hence, if the packet containing the input sequence is successfully delivered at time $t$, then the actuator will apply the vector $u_{t, t}^{*}$. If, instead, the packet is dropped, then the actuator will apply the vector $u_{t-1, t}^{*}$, and a similar procedure is repeated if consecutive actuation packets are dropped. Thus, the buffer allows us to feed the actuator with an optimal (albeit potentially obsolete) input, even if $N$ packets are consecutively dropped, as explained in Reference [22].

It is worth noting that while the state observer provides robust and theoretically sound protection against loss of sensing information, the buffers delivering samples to the actuators are implemented following a heuristic approach. Indeed, one would expect that if the MPC scheme is properly tuned and there are no external disturbances, then any two consecutive actuation signals, say $u_{t, t+1}^{*}$ and $u_{t+1, t+1}^{*}$, will not be very different. The limit case occurs when the plant reaches steady-state and no external disturbances are applied to the plant, where the MPC scheme computes $N$ identical samples every time, i.e., $u_{t, j}^{*}=\bar{u}$ for each $j$. Thus, in that case, our buffer heuristics allow us to withstand the loss of $N$ consecutive actuation packets without a control performance loss. This situation is exemplified in both simulations in Section 6. 


\subsection{Wireless Network and Manager}

We adopt a WirelessHART [17] architecture for our WSAN design, which is tailored for applications in industrial wireless process automation by selecting a set of specific network features that enable timely and highly reliable communication. A WirelessHART network is a wireless multi-hop mesh network consisting of a number of battery-powered field devices connected to a gateway through access points. The network is managed by a centralized network manager, usually collocated with the gateway. The network manager collects topology information from the field devices, computes routes and transmission schedules, and disseminates routing information and schedules among field devices.

WirelessHART adopts the IEEE 802.15.4 physical layer, designed for low cost and low data rate communication. Transmissions are scheduled based on a time-slotted channel hopping (TSCH) MAC protocol, which can provide a deterministic and collision-free communication compared to CSMA/CA, and which works perfectly with periodic communication. Each timeslot is $10 \mathrm{~ms}$, long enough to accommodate a transmission and its acknowledgement. For transmissions between pairs of nodes, a timeslot can either be dedicated or shared. In a dedicated slot, only one sender is allowed to transmit. In a shared slot, more than one sender competes for one transmission opportunity. WirelessHART networks operate on a $2.4 \mathrm{GHz}$ ISM band, and they can use up to 16 channels, as defined in the IEEE 802.15.4 physical layer standard. Also, WirelessHART networks adopt channel hopping for channel diversity, periodically changing the communication channel according to a predetermined schedule. At the network layer, the WirelessHART protocol supports two types of routings, namely, source and graph routing. Source routing provides a single (primary) path from source to destination, while graph routing consists of a single primary path and a backup path for each node on a primary path.

As explained in Section 1, the performance of the wireless network and the closed-loop control system are intertwined. Among all the statistics one can use to measure the performance of a wireless network, the packet delivery ratio (PDR) is at first sight the most significant for control applications, since a perfect PDR implies that all the information sent through the network is eventually delivered. On the other hand, a high PDR in multi-hop networks comes with long delays due to redundant transmission and route diversity, which can be longer than the information flow deadlines. Therefore, in this article, we propose a novel holistic controller, capable of balancing physical and wireless requirements while maintaining the stability of the plant. The holistic controller will simultaneously compute actuation signals and command the network to update its configuration as a function of current PDR measurements and worst-case state performance predictions.

Network reliability can be achieved through means such as packet retransmission, route diversity, or channel diversity, among others. Our design adopts a mechanism in which the number of transmissions of a certain route changes according to the conditions of the network and the physical plant. In this article, we avoid modifying more than one network configuration parameter to simplify our presentation and avoid an unnecessarily complex algorithm.

The transmission number (\#TX) is at the center of the tradeoff between reliability and network resources, i.e., more transmissions lead to a higher delivery ratio at a cost of network resources. Figure 3 depicts the relationship between end-to-end delivery ratio and the retransmission number (\#ReTx) under a 16-node WSAN. There is a diminishing return in PDR improvement as the \#ReTx increases. (The settings of this group of experiments are the same as in Section 6.)

On the other hand, higher PDRs do not immediately imply a good closed-loop performance in the physical plant, as shown in Figure 4. (The settings of this group of experiments are also the same as in Section 6.) Indeed, the internal properties of the physical plant, such as its stability, 


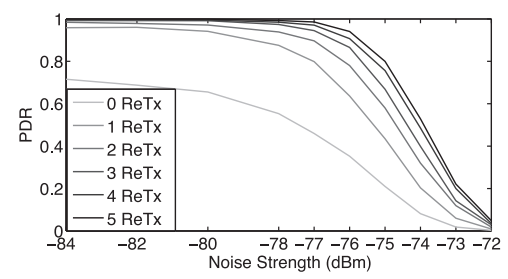

Fig. 3. Average PDR measured for different retransmission indices under different wireless background noise conditions.

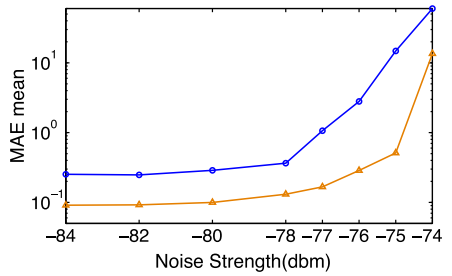

(a) Open-loop unstable plant.

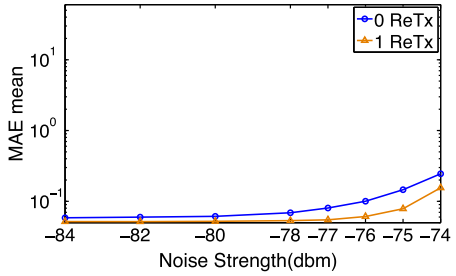

(b) Open-loop stable plant.

Fig. 4. Mean Absolute Error (MAE) for different retransmission indices under different wireless background noise conditions, evaluated on two linear systems: one open-loop unstable (a) and the other open-loop stable (b). Note how higher PDRs have different consequences depending on the properties of the physical plant.

inherently limit and shape the effect that improvements in network communication have on the final control objective. Our new holistic controller collects and intertwines information from both the physical system and wireless network, adapting all the available parameters towards the goal of stable and safe physical executions.

In this article, we aim not only to find theoretical sufficient conditions to guarantee the stability of the physical plant, but also to provide an implementable algorithm for the holistic controller and network manager.

\section{HOLISTIC CONTROLLER DESIGN}

Compared to traditional computing systems, a unique dependability challenge of cyber-physical systems is to maintain dependable control under disturbance from both the cyber and physical sides. Note that poor control performance or even system instability may lead to significant damage in a physical plant. Maintaining control performance under disturbances is therefore a fundamental dependability concern in industrial control systems. A dependable industrial WCS must be resilient against disturbances in both the wireless networks and the physical plant. The key contribution of our holistic control approach is enhancing the dependability of such systems through network adaptation based on the control performance of the physical plant, thereby integrating cyber and physical components.

As shown in Figure 5, our holistic control system maintains dependability under cyber and physical disturbances as follows: (1) wireless interferences and physical disturbances worsen physical control performance, which is reflected in an increase in the Lyapunov function in the controller; (2) the network manager generates network adaptation commands if the Lyapunov function is higher than the upper threshold; (3) each node reconfigures the number of transmissions (\#Tx) after receiving the adaptation commands from the network manager; and (4) the resulting improvement in network reliability causes the control performance to recover. 


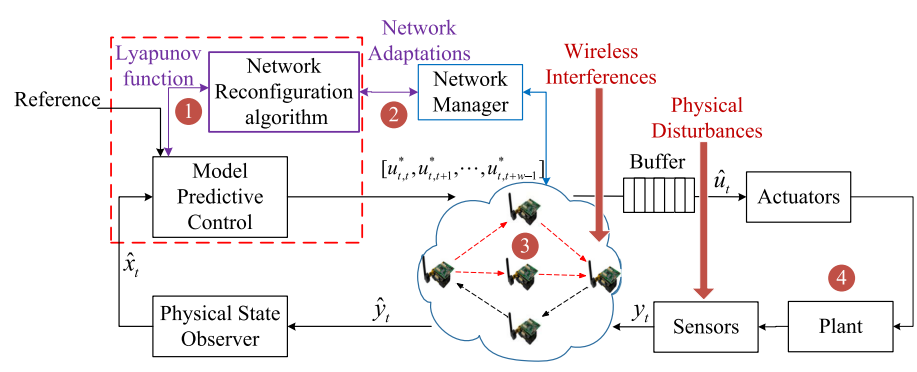

Fig. 5. Dependable architecture under cyber and physical interferences.

In this section, we focus on three key areas to achieve our objective of designing a stable controller over a WirelessHART multi-hop network. First, we show that in an ideal case, where the network delivers every packet with no delay, the MPC scheme in Equation (2) results in asymptotically stable executions. We achieve this goal by showing that the function $V(x)$, defined in Equation (2), is in fact a Lyapunov function. Second, we find theoretical bounds for the worstcase evolution of the Lyapunov function $V(x)$. Thus, if the wireless control architecture described in Section 3 results in values of the Lyapunov function that violate the worst-case bounds, then we must adjust the parameters in the wireless network to increase reliability. Third, we use this adaptation principle to build a holistic algorithm that guarantees the stability of the physical plant while simultaneously reducing the latency and power usage of the wireless network.

\subsection{Stable Control of the Physical Plant}

Our holistic controller uses a combination of theoretical guarantees and real-time observations to decide how many transmissions must schedule each node in the wireless network. At the core of our algorithm is the guarantee that, using an ideal network, the MPC scheme defined in Section 3 results in (exponentially) asymptotically stable trajectories. Without loss of generality, we assume throughout this section that $\bar{x}=0$ and $\bar{u}=0$, as defined in Equation (3), to simplify our notation.

We follow the strategies described in References [36] and [27, Sec. 3.3] to prove the stability of our MPC scheme. In particular, using the notation in Equation (2), given the matrices $Q$ and $R$, we compute $P$ as the unique positive definite solution of the following discrete-time algebraic Riccati equation:

$$
P=A^{T} P A+Q-A^{T} P B\left(R+B^{T} P B\right)^{-1} B^{T} P A,
$$

and we define

$$
K=-\left(R+B^{T} P B\right)^{-1} B^{T} P A .
$$

Lemma 4.1. Assume that $\mathcal{X}$ and $\mathcal{U}$ are polytopes, i.e., $\mathcal{X}=\left\{x \mid \Gamma_{x} x \leq b_{x}\right\}$ and $\mathcal{U}=$ $\left\{u \mid \Gamma_{u} u \leq b_{u}\right\}$, and consider the MPC scheme in Equation (2).

If $P$ is defined as in Equation (4), $K$ is defined as in Equation (5), $S=\beta P$ for $\beta \geq 1$, and

$$
\mathcal{X}_{f}=\left\{x \mid\left[\begin{array}{c}
\Gamma_{x} \\
\Gamma_{u} K
\end{array}\right] x \leq\left[\begin{array}{l}
b_{x} \\
b_{u}
\end{array}\right]\right\},
$$

then the system in Equation (1) is asymptotically stable and $V(x)$ is a Lyapunov function.

Proof. It is sufficient to show that we satisfy the conditions in Assumptions A1 to A4 in Reference [27, Sec. 3.3], where $\kappa_{f}(x)=K x$. Indeed, Assumptions A1 and A2 are trivially satisfied thanks to our definition in Equation (6). Assumption A3 follows Lemma 1 in Reference [36], and Assumption A4 is satisfied since a simple algebraic manipulation of Equation (4) implies that 


$$
(A+B K)^{T} S(A+B K)-S+Q+K^{T} R K=(1-\beta)\left(Q+K^{T} R K\right),
$$

where the right-hand side is a negative semi-definite matrix, as desired.

Note that $\beta$ allows us to easily adjust the transient response while maintaining the stability guarantee. Also note that in pathological situations, the set $\mathcal{X}_{f}$ in Equation (6) could be empty or have no interior. A discussion regarding those situations is beyond the scope of this article; we refer the interested reader to Reference [8, Ch. 5.2.3].

Our holistic controller uses the value of the Lyapunov function $V\left(\hat{x}_{t}\right)$ to test if the wireless network has an undesired impact on the performance of the physical plant. Our test requires calculating three parameters, $\left\{\alpha_{i}\right\}_{i=1}^{3}$, as explained below.

Lemma 4.2. Consider $V(x)$ as defined in Equation (2). Let $P_{0}$ be a positive definite matrix computed recursively via the time-varying discrete-time Riccati equation:

$$
P_{k-1}=A^{T} P_{k} A+Q-A^{T} P_{k} B\left(R+B^{T} P_{k} B\right)^{-1} B^{T} P_{k} A,
$$

with $P_{N}=S$, and with $\alpha_{1}$ as the smallest eigenvalue of $P_{0}$. Then $V(x) \geq \alpha_{1}\|x\|^{2}$.

Proof. If we relax the optimization problem by setting $\mathcal{U}=\mathbb{R}^{m}$ and $\mathcal{X}=\mathcal{X}_{f}=\mathbb{R}^{n}$, then we obtain a finite-horizon LQR problem. As explained in Reference [5, Ch. 4.1], the value of a finitehorizon LQR problem with an initial condition of $x$ is $x^{T} P_{0} x$; hence $V(x) \geq x^{T} P_{0} x \geq \alpha_{1}\|x\|^{2}$.

Note that if we choose $\beta=1$, then $P_{0}=P$, as defined in Equation (4); thus we simplify the numerical calculation of $\alpha_{1}$.

Lemma 4.3. Consider $V(x), A, Q$, and $S$ as defined in Equation (2). Let

$$
M=\sum_{j=0}^{N-1}\left(A^{j} Q A^{j}\right)+A^{N} S A^{N},
$$

and let $\alpha_{2}$ be the largest eigenvalue of $M$. Then $V(x) \leq \alpha_{2}\|x\|^{2}$.

Proof. The proof follows directly after noting that $u_{j}=0$ for each $j$ is a feasible input signal. Thus, $V(x) \leq x^{T} M x \leq \alpha_{2}\|x\|^{2}$.

Lemma 4.4. Consider the system in Equation (1) with the closed-loop controller in Equation (2). Let $\alpha_{3}$ be the smallest eigenvalue of $Q$. Then $V\left(x_{t+1}\right)-V\left(x_{t}\right) \leq-\alpha_{3}\left\|x_{t}\right\|^{2}$.

Proof. Let $\left\{u_{t, j}^{*}\right\}_{j=t}^{t+N-1}$ be the optimal input signal associated with the value function $V\left(x_{t}\right)$. Let $\left\{x_{j}\right\}_{j=t}^{t+N}$ be the execution resulting from applying the input $\left\{u_{t, j}^{*}\right\}_{j=t}^{t+N-1}$.

Note that the input signal $\left\{u_{t, t+1}^{*}, u_{t, t+2}^{*}, \ldots, u_{t, t+N-1}^{*}, K x_{t+N}\right\}$ belongs to the feasible set of the problem with value $V\left(x_{t+1}\right)$. Hence,

$$
V\left(x_{t+1}\right) \leq \sum_{j=t+1}^{t+N-1}\left(x_{j}^{T} Q x_{j}+\left(u_{t, j}^{*}\right)^{T} R u_{t, j}^{*}\right)+x_{t+N}^{T}\left(Q+K^{T} R K+(A+B K)^{T} S(A+B K)\right) x_{t+N},
$$

and

$$
V\left(x_{t+1}\right)-V\left(x_{t}\right) \leq-x_{t} Q x_{t} \leq-\alpha_{3}\left\|x_{t}\right\|^{2},
$$

where we use the result in Equation (7) after eliminating all the repeated terms in Equation (10) and $V\left(x_{t}\right)$. 
Using the parameters $\left\{\alpha_{i}\right\}_{i=1}^{3}$, we now obtain two results that will become the test conditions to evaluate the performance loss of the physical control loop due to information loss in the wireless network.

Lemma 4.5. Consider the system in Equation (1) with the closed-loop controller in Equation (2). If $V(x) \leq \alpha_{1} \gamma$, then $\|x\|^{2} \leq \gamma$.

Proof. Suppose that $\|x\|^{2}>\gamma$, then $V(x) \geq \alpha_{1}\|x\|^{2}>\alpha_{1} \gamma$. The result follows, using the contrapositive of the argument above.

Lemma 4.6. Consider the system in Equation (1) with the closed-loop controller in Equation (2). Then, for each $j \in \mathbb{N}$,

$$
V\left(x_{t+j}\right) \leq\left(1-\frac{\alpha_{3}}{\alpha_{2}}\right)^{j} V\left(x_{t}\right) .
$$

Proof. Using the results in Lemmas 4.3 and 4.4 we get that, for each $t \in \mathbb{N}, V\left(x_{t+1}\right) \leq(1-$ $\left.\frac{\alpha_{3}}{\alpha_{2}}\right) V\left(x_{t}\right)$. The desired result follows by induction.

\section{2 \#Tx Adaptation}

As explained in Section 3, among all the configuration parameters of the wireless network that we can modify, we have chosen to adapt the \#Tx, denoted $\eta_{t}$, that each node in the network uses to determine the maximum number of retries used to send a packet before it is dropped. While one can intuitively expect that more transmissions should improve the physical control loop performance, they come associated with longer delays and shorter battery lifetimes for the nodes. Moreover, it is not immediately clear how to quantify the impact that more transmissions have in the control loop, as shown in Figure 4.

The value of the Lyapunov function $V\left(\hat{x}_{t}\right)$ at each $t$ for each control loop, together with the bounds in the lemmas above, give us a constructive testing mechanism to evaluate the impact that the loss of information in the WSN has on the physical plant. Suppose that a setpoint $\bar{x}$ has been computed as in Equation (3), and a desired maximum deviation from that setpoint, $\gamma$, has been defined, i.e., the goal is to maintain $\left\|x_{t}-\bar{x}\right\|^{2} \leq \gamma$ for each $t \in \mathbb{N}$. To build a \#Tx adaptation algorithm based on the analytical results above, we must first establish a set of principles that our algorithm must satisfy:

(1) If $V\left(x_{t}\right) \leq \alpha_{1} \gamma$, then $\eta_{t+1} \leq \eta_{t}$, i.e., $\eta_{t}$ will not increase, since the physical plant is within acceptable bounds, as shown in Lemma 4.5 .

(2) Given $\lambda \in(0,1)$, if $V\left(x_{t}\right) \geq \lambda \alpha_{1} \gamma$, then $\eta_{t+1} \geq \eta_{t}$, i.e., $\eta_{t}$ will not decrease, since the physical plant might approach the safety bound in Lemma 4.5.

(3) If $V\left(x_{t+j}\right) \leq\left(1-\frac{\alpha_{3}}{\alpha_{2}}\right)^{j} V\left(x_{t}\right)$, then $\eta_{t+j} \leq \eta_{t}$, i.e., $\eta_{t}$ will not increase, since the physical plant is evolving towards its equilibrium point within expected bounds, as shown in Lemma 4.6.

(4) If the current PDR, denoted $\rho_{t}$, is below a threshold, say $\rho_{t}<\rho_{\min }$, then $\eta_{t+1} \geq \eta_{t}$, i.e., $\eta_{t}$ will not decrease, since the network must maintain a minimum connectivity level.

The parameter $\lambda$ is used to create a dead-band between increases and decreases of the \#Tx. Indeed, if $V\left(x_{t}\right) \in\left[\lambda \alpha_{1} \gamma, \alpha_{1} \gamma\right]$ then $\eta_{t}$ remains constant. Our \#Tx adaptation algorithm for each control loop is described in detail in Algorithm 1. Given a control loop, we measure the current PDR $\rho_{t}$ on each iteration, and we compute $V\left(\hat{x}_{t}\right)$. The variable $\delta$ is used as an internal state to determine whether the last \#Tx change was an increase $(\delta=1)$ or a decrease $(\delta=0)$. The parameter $\lambda \in(0,1)$ determines the width of the dead-band for the Lyapunov function when \#Tx is left unchanged. The parameter $\tau_{1} \in \mathbb{N}$ is the minimum number of iterations it takes the wireless network to propagate 


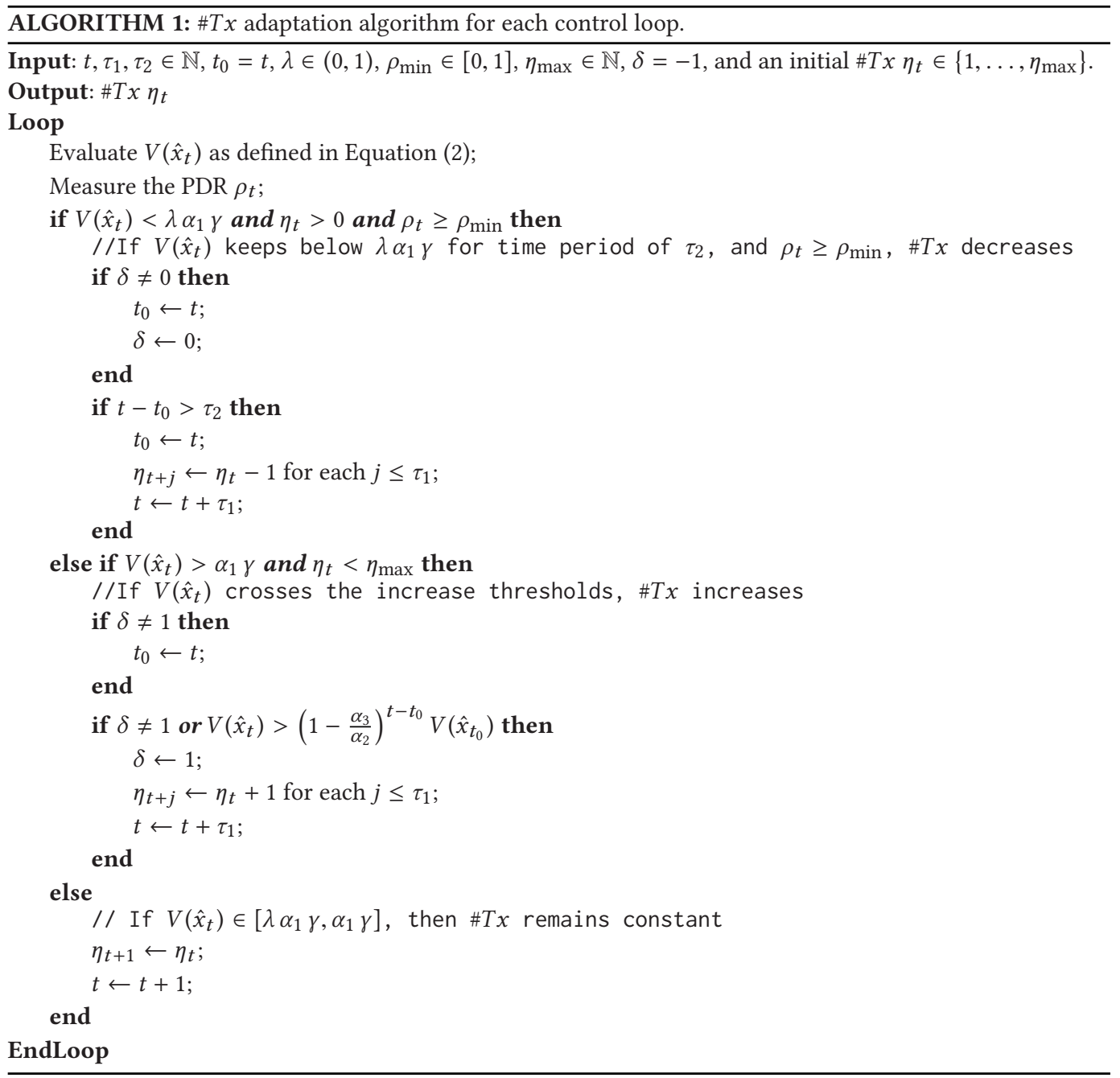

the new transmission schedule, as explained in Section 5. The parameter $\tau_{2} \in \mathbb{N}$ is used to slow the rate of the \#Tx decreases, since those might eventually result in violations of the safety bound in Lemma 4.5. Finally, the parameters $\eta_{\max }$ and $\rho_{\min }$ are chosen such that all routes can be scheduled and minimum network control information can still be delivered. Since the calculation of $V\left(\hat{x}_{t}\right)$ is necessary for MPC to compute the control command for the actuator, the task of the algorithm is to compare the $\rho_{t}, V\left(\hat{x}_{t}\right)$ with its thresholds and decide whether to decrease, increase, or maintain the \#Tx. Therefore, Algorithm 1 is lightweight, with $O(1)$ complexity.

Our control strategy cannot mathematically guarantee the closed-loop system stability of the WSAN unless extra assumptions are considered, which is a common approach in the literature [1, 15, 25]. The applicability and appropriateness of these assumptions depend on the particular properties of the industrial plant at hand, thus we avoid imposing a particular framework in this article. Instead, our algorithm takes a best-effort approach towards balancing closed-loop performance and network load, which is a practical heuristic in real-world scenarios. The stability 
Table 1. Piggyback Algorithm Superframe Examples

\begin{tabular}{|c|c|c|c|c|c|c|c|c|c|c|c|c|}
\hline$\# T x$ & Slot 1 & Slot 2 & Slot 3 & Slot 4 & Slot 5 & Slot 6 & Slot 7 & Slot 8 & Slot 9 & Slot 10 & Slot 11 & Slot12 \\
\hline 1 & $\mathrm{~A} \rightarrow \mathrm{B}$ & & & $\mathrm{B} \rightarrow \mathrm{C}$ & & & $\mathrm{A} \rightarrow \mathrm{B}$ & & & $\mathrm{B} \rightarrow \mathrm{D}$ & & \\
\hline 2 & $\mathrm{~A} \rightarrow \mathrm{B}$ & $\mathrm{A} \rightarrow \mathrm{B}$ & & $\mathrm{B} \rightarrow \mathrm{C}$ & $\mathrm{B} \rightarrow \mathrm{C}$ & & $\mathrm{A} \rightarrow \mathrm{B}$ & $\mathrm{A} \rightarrow \mathrm{B}$ & & $\mathrm{B} \rightarrow \mathrm{D}$ & $\mathrm{B} \rightarrow \mathrm{D}$ & \\
\hline 3 & $\mathrm{~A} \rightarrow \mathrm{B}$ & $\mathrm{A} \rightarrow \mathrm{B}$ & $\mathrm{A} \rightarrow \mathrm{B}$ & $\mathrm{B} \rightarrow \mathrm{C}$ & $\mathrm{B} \rightarrow \mathrm{C}$ & $\mathrm{B} \rightarrow \mathrm{C}$ & $\mathrm{A} \rightarrow \mathrm{B}$ & $\mathrm{A} \rightarrow \mathrm{B}$ & $\mathrm{A} \rightarrow \mathrm{B}$ & $\mathrm{B} \rightarrow \mathrm{D}$ & $\mathrm{B} \rightarrow \mathrm{D}$ & $\mathrm{B} \rightarrow \mathrm{D}$ \\
\hline
\end{tabular}

of our algorithm hinges on the relation between dropped actuation packets and their impact on the empirical Lyapunov function at each iteration, which has been studied in the past $[2,28]$.

\section{NETWORK RECONFIGURATION}

In this section, we introduce a practical runtime reconfiguration protocol for the WirelessHART network. To facilitate changing the number of transmissions per hop at runtime, the centralized scheduler on the network manager reserves enough slots to accommodate a transmission and the maximum number of retransmissions for each intended transmission over a link. This additional constraint can be incorporated in any scheduling policy. According to the WirelessHART standard, we enable packet retransmissions for links on the primary path for both source and graph routings. Specifically, when the scheduler schedules transmissions over each link, it should reserve $\eta_{\max }$ dedicated slots, i.e., we first reserve a timeslot for packet transmission, and then allocate $\eta_{\max }-1$ slots for its retransmissions, where $\eta_{\max }$ is the maximum number of transmissions per hop.

Our previous research has demonstrated that a WCS can have different levels of resilience to packet loss for sensing and actuation. Motivated by the asymmetric routing idea in Reference [22], we develop an asymmetric scheduling approach in which the number of packet transmissions of the sensing and actuation phase can be configured independently. Considering that sensing data are less vulnerable to packet loss because of the state observer, we do not allocate retransmissions for sensing packets. However, we allow the holistic controller to adaptively adjust the number of transmissions for actuation packets of each control loop, based on the physical and network conditions. This need for adjustment stems from the fact that the control performance is more sensitive to packet loss from the controller to the actuators, despite the buffered control inputs.

We next present a runtime transmission adaptation protocol. As already explained, the network manager generates a schedule that allocates enough slots to accommodate the maximum \#Tx over each link defined by the $T x$ adaptation algorithm. Each schedule entry is represented by a tuple [slot_offset, channel, sender, receiver, flowID, \#Tx(flowID)]. A transmission schedule is called a superframe, and repeats itself during runtime. The slot_offset is the relative timeslot number in a superframe. The flowID specifies the flow a transmission belongs to, and \#Tx(flowID) indicates the current \#Tx of this flow, enabling the protocol to configure \#Tx of each flow independently. In Table 1, 1Tx to 3Tx presents a TDMA-schedule for two flows, $F 1$ and $F 2$, that deliver data through nodes $\mathrm{A} \rightarrow \mathrm{B} \rightarrow \mathrm{C}$ and $\mathrm{A} \rightarrow \mathrm{B} \rightarrow \mathrm{D}$, respectively, when \#Tx(F1) and \#Tx(F2) vary from one to three transmissions. The superframe has a length of 12 timeslots. Note that other routing and scheduling algorithms optimize network resource usage to enhance network scalability, but these are not within the scope of this work.

We adopt a piggyback mechanism to disseminate a newly computed \#Tx for a certain control loop (flow) generated by the holistic controller. A network manager, which is co-located with a holistic controller, can incorporate the updated \#Tx and the \#Flow into all the periodic actuation packets in this control loop. Hence, all nodes along the actuation routes of certain control loops can receive this update. This piggyback mechanism helps reduce communication cost by utilizing existing periodic communication. Once a node receives a \#Tx switch command, it will apply a new $\# T x$ at the beginning of the next superframe. 
However, if a node fails to receive the command due to packet loss, it will continue to use the current \#Tx until any actuation packet is received. Therefore, it is possible that, at the same time, different nodes along the route of a flow may use different \#Tx. Nevertheless, it is still possible for nodes to eventually receive the update since they are always scheduled to communicate at the slot allocated for their first transmission attempts over a link. For example, in Table 1, the transmissions colored red represent the current schedule for flow F1 $(\mathrm{A} \rightarrow \mathrm{B} \rightarrow \mathrm{C})$ and F2 $(\mathrm{A} \rightarrow \mathrm{B} \rightarrow \mathrm{D})$, when a controller issues a command to update \#Tx(F1) from 1 to 2 , and \#Tx $(\mathrm{F} 2)=3$. In this example, the update reaches node $\mathrm{A}$ and $\mathrm{B}$ at timeslot 1 , but fails to arrive at $\mathrm{C}$ at timeslot 4 due to packet loss. Hence, $\mathrm{A}$ and $\mathrm{B}$ switch to $2 T x$, while $\mathrm{C}$ continues to use $1 T x$. Although B and C use different \#Tx, it is still possible for $\mathrm{C}$ to receive actuation and mode switch commands from $\mathrm{B}$ in the following superframe, since B and C will always communicate at slot 4 regardless of \#Tx. The \#Tx of F2 is unaltered during the process since there is no \#Tx update for F2.

The dependability of the holistic management system should also be considered. According to WirelessHART standard, a network can handle failures in a network manager through replication [39]. Specifically, a network can support redundant network managers. However, there must be one and only one active network manager per network. The active network manager is responsible for keeping the standby network managers synchronized. Switchover between network managers may be initiated by a fault detection mechanism or manually initiated. In a holistic control system, the holistic controller and network manager will be replicated in a similar fashion to the current WirelessHART standard to handle failures in the network management system.

It is worth noting that our article mainly discusses WSANs that are revivable under moderate cyber and physical attacks. Thus we set $\rho_{\min }$ to guarantee relatively high PDRs of wireless networks in Section 4.2. Under extreme conditions, a larger portion of piggyback packets may be lost. In this case, sending commands to switch modes by broadcasting or flooding might be a better solution.

\section{CASE STUDY}

In this section, we introduce a systematic case study for our holistic WSAN controller. It is extremely challenging to conduct experiments on industrial control systems in the field, especially under cyber and physical disturbances. Small lab-scale equipment, on the other hand, is usually too small for realistic wireless experiments. To overcome this challenge, we developed WCPS, an open-source simulator that integrates realistic simulations of both wireless networks and physical plants. WCPS integrates Simulink and TOSSIM, a state-of-the-art sensor network simulator featuring a realistic wireless model seeded by real-world wireless traces [22-24]. Both Simulink and TOSSIM are among the leading simulators in the control and networking communities, respectively. Furthermore, the WirelessHART network protocol stack is implemented as part of WCPS 2.0 [23], including protocols at the routing (Source and Graph Routing) and MAC layers. To the best of our knowledge, WCPS is the first simulator that supports all these WirelessHART features with a realistic wireless link model.

In this article, we have implemented the runtime \#Tx reconfiguration mechanism presented in Section 5 in WCPS 4.0. Additionally, we have included multi-rate functionality in the simulation. In practice, industrial plant models mostly operate continuously or at very high frequencies. However, the wireless communication and controller execute at a relatively low frequency because of the network latency and computational latency. Therefore, we incorporate multi-rate functionality in WCPS, which simulates the plant, wireless network, and controller operating at multiple rates. Additionally, we have simulated the computational and communicational latency of each module in the wireless control loop.

On the physical side, to represent an industrial process system, we use two five-state linear time-invariant plants that share the same WSAN. One of the plants is open-loop unstable, and 
the other is open-loop stable. We also present the case study results of a realistic load positioning system in order to show the generality of our algorithm [37]. On the cyber side, we simulate a 16node WSAN using the WCPS simulator [10], seeded with real-world wireless traces as explained in References [22, 23]. Besides studying the behavior of the algorithms presented in Sections 4 and 5 , we also test the performance of our system under both cyber and physical disturbances. We will present a case study of the open-loop unstable plant in the first four subsections. Then, in Section 6.5, to form a multi-loop simulation, we will include an open-loop stable plant that shares a WSAN with that open-loop unstable plant.

\subsection{Experimental Setting}

The plant is defined in Equation (1), with the following parameters:

$$
A=\left[\begin{array}{ccccc}
0.717 & -1.367 & -0.218 & -0.867 & -0.899 \\
0.078 & 0.209 & -0.105 & -0.511 & -0.466 \\
0.122 & 0.891 & 1.305 & 0.511 & 0.666 \\
-0.243 & -1.383 & -0.610 & -0.023 & -0.932 \\
0.122 & 0.871 & 0.165 & 0.712 & 1.466
\end{array}\right], \quad B=\left[\begin{array}{c}
0.083 \\
0.056 \\
-0.056 \\
0.111 \\
-0.056
\end{array}\right], \quad \text { and } \quad C=\left[\begin{array}{l}
0 \\
1 \\
1 \\
0 \\
0
\end{array}\right]^{T} \text {. }
$$

Note that the set of eigenvalues of $A$ is equal to $\{0.413,0.563,0.624,1.068,1.006\}$. Since there are two eigenvalues outside the unit circle, the plant is open-loop unstable. The parameters of the MPC scheme in Equation (2) are chosen as follows:

$$
\begin{gathered}
\left.Q=\left[\begin{array}{lllll}
1 & 0 & 0 & 0 & 0 \\
0 & 1 & 0 & 0 & 0 \\
0 & 0 & 1 & 0 & 0 \\
0 & 0 & 0 & 5 & 0 \\
0 & 0 & 0 & 0 & 1
\end{array}\right], \quad S=\begin{array}{ccccc}
439 & 518.37 & 942.57 & 220.13 & 588.16 \\
518.37 & 633.85 & 1124.8 & 266.03 & 708.54 \\
942.57 & 1124.8 & 2045.3 & 483.1 & 1274.2 \\
220.13 & 266.03 & 483.1 & 129.01 & 309.01 \\
588.16 & 708.54 & 1274.2 & 309.01 & 809.37
\end{array}\right], \quad \bar{x}=\left[\begin{array}{c}
0.289 \\
1.735 \\
0.578 \\
-1.157 \\
-1.735
\end{array}\right], \\
K=\left[\begin{array}{lllll}
-20.49 & -20.48 & -42.66 & -11.24 & -26.38
\end{array}\right] .
\end{gathered}
$$

Also, $\bar{u}=0.2, R=0.08, N=50, \mathcal{X}=\mathbb{R}^{5}$, and $\mathcal{U}=\{|u| \leq 40\}$, where $S, K$, and $\mathcal{X}_{f}$ are computed as described in Lemma 4.1 with $\beta=1.1$. The parameters of Algorithm 1 are $\lambda=0.1, \tau_{1}=5, \tau_{2}=$ $500, \gamma=16, \alpha_{1}=1.977, \alpha_{2}=8.223 \cdot 10^{6}$, and $\alpha_{3}=1$, where $\left\{\alpha_{i}\right\}_{i=1}^{3}$ are computed as described in Lemmas 4.2 to 4.4 .

We upgrade WCPS [10] as a platform for holistic WCS simulations. WCPS employs a federated architecture that integrates Simulink [41] for simulating the physical system dynamics and controllers, and TOSSIM [21] for simulating WSANs. In this article, we have incorporated the runtime \#Tx reconfiguration mechanism, multi-rate functionality, and simulation of computation and communication delays into WCPS 4.0. Since sensing measurements and control commands are sent via WSAN periodically, time-driven scheduling is adopted, as is shown in Figure 6. We determine the length of each event based on its worst-case execution time.

We simulate a wireless network consisting of 16 nodes, where each simulation is based upon data traces collected from our WSAN testbed at Washington University. The collected information includes the received signal strength indicator (RSSI) and electromagnetic noise, which are used as inputs for the wireless link model in WCPS. The WSAN in this study consists of six sensing flows and two actuation flows. A sensing flow delivers sensing data from a sensor node to the controller, while an actuation flow delivers control commands to an actuator. Given pairs of sources and destinations, we adopt the strategy of lifetime-maximization source routing presented in Reference [45], which prolongs the network lifetime while it preserves reliability. We use only two pairs of sensing and actuation flows for two control loops. The extra sensing flows are reserved 


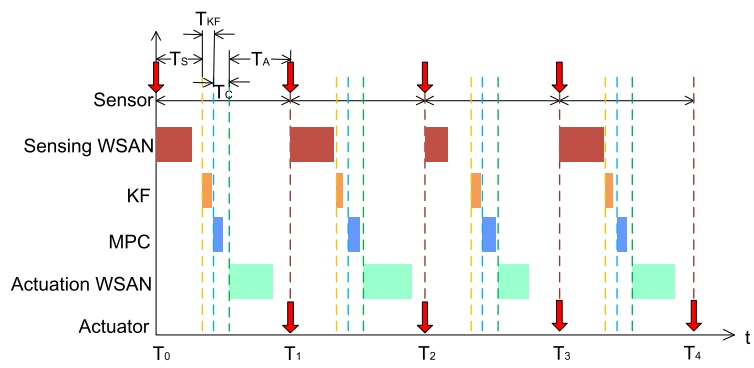

Fig. 6. Time-driven scheduling of wireless control system.

for redundant measurements, as it is commonly designed in industrial scenarios. The maximum distance from a sensor to an actuator is four hops.

Since WirelessHART employs the TSCH MAC, we schedule those eight flows in the order of flowID under the constraints of avoiding transmission conflict and channel contention in channels 22 and 23. A transmission conflict occurs when a node becomes a common node as sender or receiver in each timeslot. Channel contention occurs when all channels are assigned to other transmissions in a certain timeslot. The resultant superframe length of this WSAN is $140 \mathrm{~ms}$, i.e., 14 fixed timeslots. The WSAN sensing delay $\left(T_{S}\right)$ and actuation delay $\left(T_{A}\right)$ are set to $60 \mathrm{~ms}$ and $80 \mathrm{~ms}$, respectively. The worst-case execution times of the controller and the state observer are $30 \mathrm{~ms}$ and $0.2 \mathrm{~ms}$ among 10,000 operations, respectively. Hence, we set the computation delay of the controller $\left(T_{C}\right)$ to $30 \mathrm{~ms}$, and the Kalman Filter delay $\left(T_{K F}\right)$ to $10 \mathrm{~ms}$, because the granularity of our simulation is $10 \mathrm{~ms}$. Therefore, we set the frequencies of the WSAN, the MPC controller, and the KF state observer in our simulations to $5 \mathrm{~Hz}$, and the frequencies of the plants to $100 \mathrm{~Hz}$. Note that the control command executed at time $T_{k}$ is based on the sensor measurements at $T_{k-1}$, as is shown in Figure 6. Since we are using MPC, at time $T_{k}$, we use $\left\{u_{j, k}^{*}\right\}_{j=k-N}^{k-1}$. In this way, we mitigate the delay of all the modules and the effects of the actuation packet loss in the wireless control loop.

Because of the asymmetric resiliency of the sensing and actuation sides [22], we adopt an asymmetric scheduling strategy. For sensing flows, we do not provide any packet retransmission since the state observer mitigates the impact of packet loss, as explained in Section 3. For actuation flows, we set the maximum \#Tx to $\eta_{\max }=4$. As explained in Section 3.2, there is a diminishing return of PDR improvements as \#Tx increases. Therefore, we set $\eta_{\max }=4$ to efficiently improve $\mathrm{PDR}$ at reasonable cost. As a reasonable figure, we set the minimum PDR to $\rho_{\min }=0.7$.

\subsection{Simulation under Wireless Interference}

We consider a disturbance on the wireless network caused by an increase in background noise on all channels, which is common when a wireless sensor network is under wide-band continuous jamming attack. In this case, the channel hopping and channel blacklisting functionality of the WirelessHART standard might fail. Our simulation considers an increased value for the background noise for the first $70 \mathrm{~s}$ of $-75 \mathrm{dBm}$, and a reduction to a standard value for the rest of the simulation of $-78 \mathrm{dbm}$, as shown in Figure 7(c). Note that the physical plant is unstable and in a transient state for the first 70s; hence a low actuation PDR would likely lead to diverging trajectories.

In this simulation (Figure 7), there are two interesting observations. First, the \#Tx is increased twice, first at $t=0 \mathrm{~s}$, and again at $t \approx 33.2 \mathrm{~s}$. The first increase is due to a violation of the safety bound condition in Lemma 4.5, while the second is due to a violation of the worst-case Lyapunov evolution in Lemma 4.6. By time $t \approx 80 \mathrm{~s}$, the physical plant is well below the safety bound, implying that Algorithm 1 successfully adapted to the higher wireless background noise. Second, once the background noise is reduced, our algorithm slowly decreases the \#Tx, finally stabilizing 


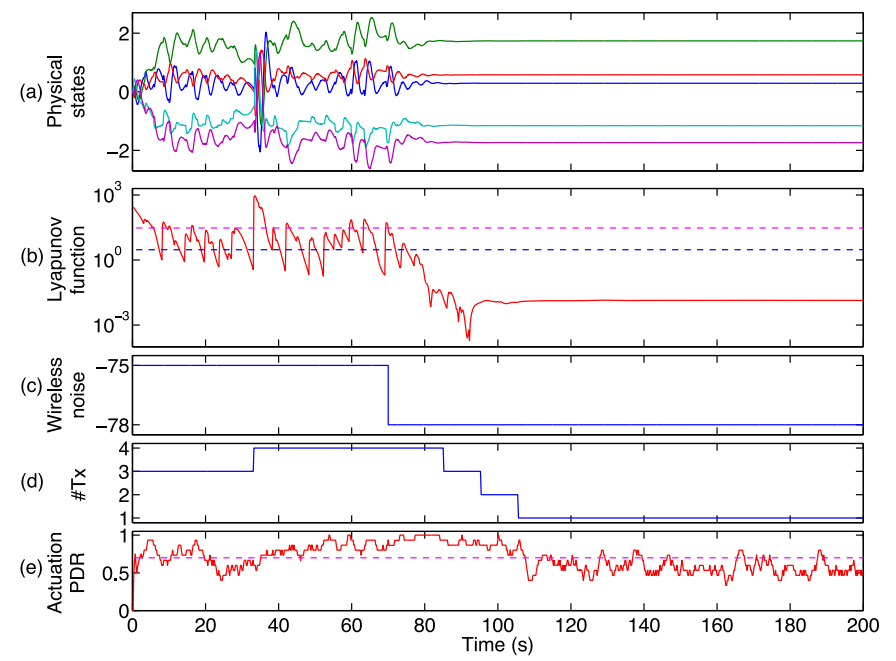

Fig. 7. Holistic controller simulation under wireless interference. The wireless background noise is higher for the first 70 s, resulting in a \#Tx adaptation.

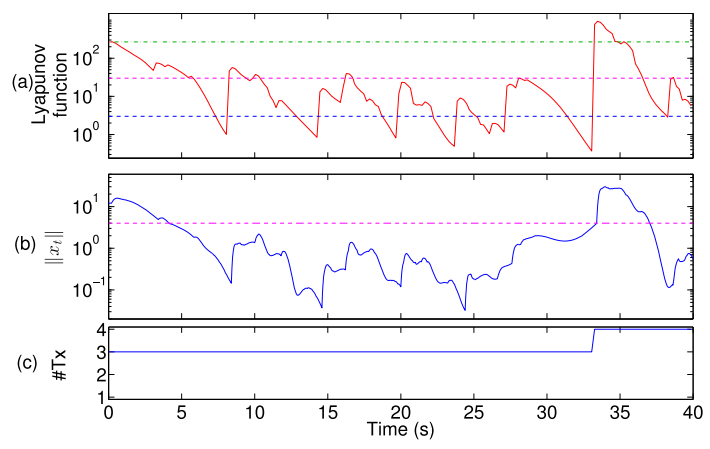

Fig. 8. Zoom in of the simulation in Figure 7 for the time interval $[0,40] \mathrm{s}$.

at a point where the physical plant is stable even under a significant number of actuation packet drops. Note that once the physical plant reaches a steady state, the optimal input sent through the network is almost constant, with $u_{t, j}^{*} \approx \bar{u}$ for each $j$. In this case, the actuation buffer, explained in Section 3 and Figure 2, almost completely mitigates the information lost in the wireless network due to lower \#Tx.

Figure 8 is a zoomed-in view of the first 40 seconds in Figure 7. Figure $8(\mathrm{a})$ and $8(\mathrm{~b})$ validate our result in Lemma 4.5 of using the Lyapunov function as a simple and practical test for safety. Also, the top green dashed line in Figure 8(a) is the bound induced by the worst-case evolution of the Lyapunov function, as explained in Lemma 4.6, which results in an increase in the \#Tx when violated.

We compare our holistic control approach against two existing approaches. In each boxplot in Figures 9, 13, and 16, from left to right, we present the results of five sets of simulations under the different management approaches:

$-\mathrm{HC}$ : our holistic control approach.

$-\mathrm{PN}$ : the physical plant and the network are controlled separately. In this traditional approach, the controller controls the physical plant, while the network manager adapts the 


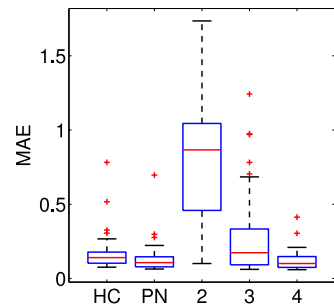

(a) Mean Absolute Error (MAE).

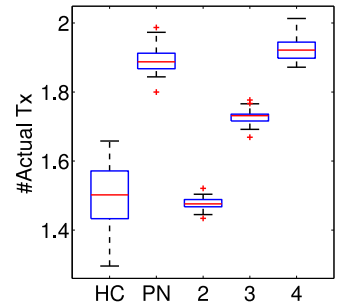

(c) Average number of actual Txs per packet.

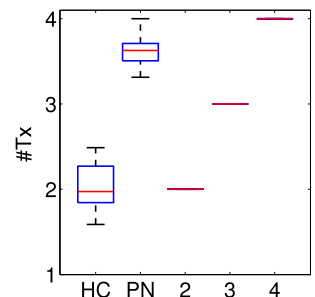

(b) Average number of scheduled Txs.

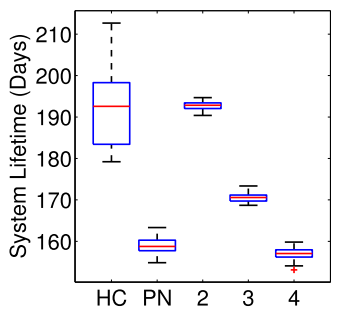

(d) Battery life.

Fig. 9. (a) MAE, (b) average \#Tx, (c) average \#Actual Tx, and (d) battery life for different \#Tx adaptation algorithms under wireless interference. The algorithms are our holistic controller (HC), pure network adaptation (PN), and constant transmission numbers equal to 2, 3, and 4, respectively.

number of transmissions based on network reliability without considering control performance. The network adaptation algorithm changes the number of transmissions (\#Tx) based on two PDR thresholds, namely, the \#Tx increase threshold (80\%) and the \#Tx decrease threshold (90\%). The network manager increases \#Tx by 1 when the PDR drops below the increase threshold, and reduces \#Tx by 1 when the PDR reaches $90 \%$.

$-2 T x, 3 T x, 4 T x$ : fixed numbers of transmissions, at 2, 3, and 4, respectively. Existing WSANs typically employ a fixed number of transmissions.

In all experimental settlings, we adopt the same physical control design based on the buffered MPC explained in Section 3.1. If an actuation packet adopts 1 Tx, the average PDR is around 23\% under a noise level of $-75 \mathrm{dbm}$, and around $53 \%$ even under a noise level of $-78 \mathrm{dbm}$, which will damage system performance. Thus, we set at least one retransmission for actuation packets. Each algorithm is simulated 40 times, and unstable executions are discarded to avoid distorting the average computations. It is worth noting that fixed $2 T x$ s results in 10 unstable simulations, and both PN and fixed 3, 4 Txs result in 1 unstable simulation, while HC stabilizes all the executions.

Figure 9 compares (a) the mean absolute error (MAE) of the physical states, (b) the number of scheduled transmissions (\#Tx for short in the rest of the article), (c) the average number of actual transmissions per actuation packet (\#Actual Tx for short in the rest of the article), and (d) the system lifetime with various wireless network configuration methods. In reality, \#Actual Tx is often less than scheduled \#Tx. For example, if the sender gets the acknowledgement from the receiver at the first try, it will not send an extra time since the packet has already been received. As another example, if the packet is lost in previous hops, the sender has no packet to send. The \#Actual Tx is one determinant of battery life. We assume wireless motes use AA batteries, with a capacity of $8640 \mathrm{~J}$. We define the system lifetime as the battery life of the most consuming node in the network, and calculate battery life based on the method in Reference [46]. According to 


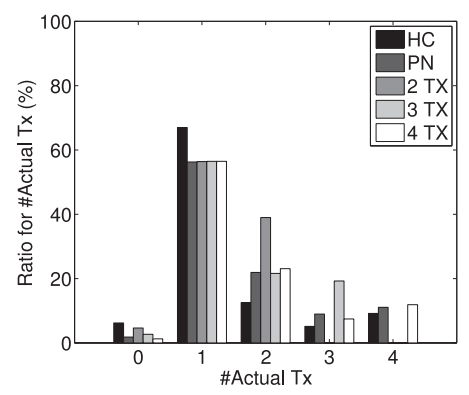

Fig. 10. Ratio for \#Actual Tx under wireless interference.

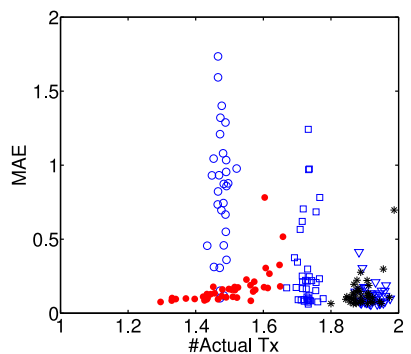

(a) Relationship between MAE and \#Actual Tx.

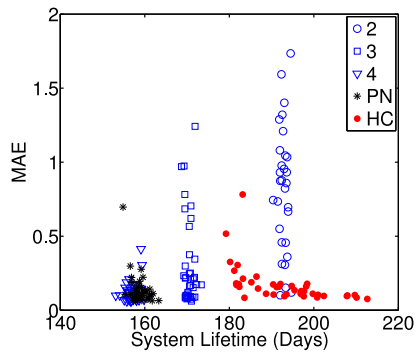

(b) Relationship between MAE and battery life.

Fig. 11. Relationship between MAE and \#Actual Tx as well as system lifetime for different \#Tx adaptation algorithms under wireless interference. The algorithms are HC (red), PN (black), and fixed \#Tx (blue), respectively.

Figure 9(a) and 9(b), while our HC algorithm has a comparable MAE to PN and 4 Txs, its average scheduled \#Tx is around 2. Figure 10 shows the ratio for \#Actual Tx with various wireless network configuration methods. $\mathrm{HC}$ has the highest ratio of 0 and 1 actual $T x$ per packet, since the system performances are sometimes acceptable even though the PDR is not high enough. At the same time, $\mathrm{HC}$ can also adjust the \#Actual Tx to 4 to guarantee control performance when needed. Figure 9(c) and 9(d) show that the \#Actual Tx and the system lifetime of our HC algorithm are also similar to $2 T x$. Furthermore, HC extends the system lifetime for more than 1 month over PN.

Figure 11 compares the relationship between MAE and the \#Actual Tx, as well as the system lifetimes for different network configuration algorithms. HC's data points are concentrated in the bottom left area of Figure 11(a), which indicates that this algorithm acquires smaller MAE with fewer \#Actual Tx. The simultaneous increase of both MAE and \#Actual Tx can be explained by the intuition of $\mathrm{HC}$ that poorer system performances will cause an increase of \#Actual Tx. On the other hand, no extra transmissions will be adopted when the physical system is in a good condition. This trend indicates that network resources are adapted well, based on the status of the physical plant. The same facts are reflected in Figure 11(b).

\subsection{Simulation under Sensor Disturbance}

We now consider an external bias applied to the physical plant sensor, modeling either a malfunction or an adversarial attack. That is, we consider a system where the output Equation (1) becomes $y_{t}=C x_{t}+\omega_{t}$ for $\omega_{t} \in \mathbb{R}$. In this simulation, we keep the wireless background noise constant at an arbitrary $-76 \mathrm{dBm}$. 


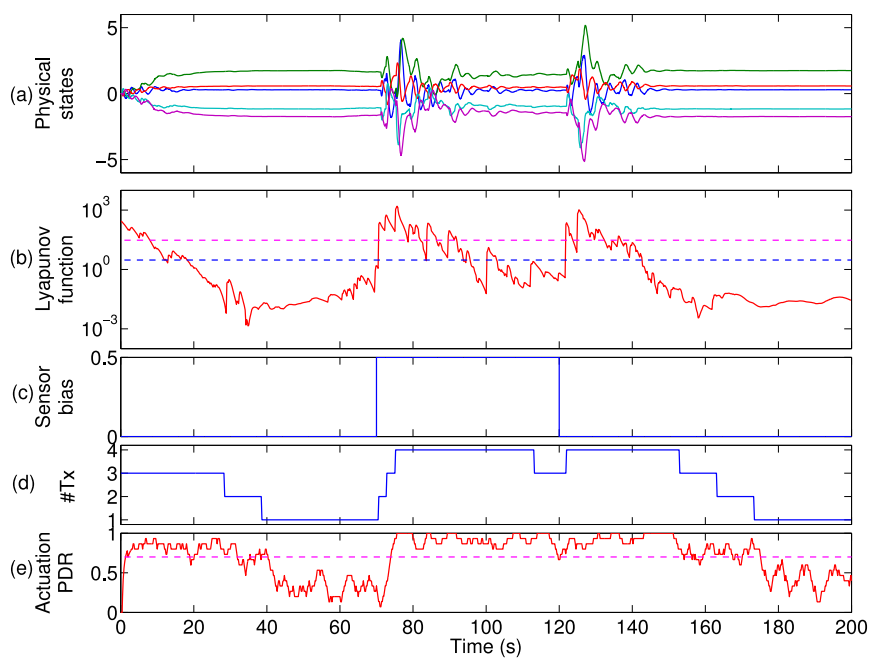

Fig. 12. Holistic controller simulation under physical disturbance.

Figure 12 shows the results of our simulation, where a disturbance is applied in the interval $[70,120] \mathrm{s}$, with $\omega_{t}=0.5$. Again, we focus our attention on two outcomes of this simulation. First, our algorithm twice lowers the \#Tx to 1, while maintaining the stability of the physical plant. Note that the actuation PDR in both situations is well below standard acceptable values, yet the use of an actuation buffer mitigates any significant impact that the information loss has in the physical plant. Second, the plant remains stable under the application and later release of the physical disturbance, both times thanks to an increase in the \#Tx. This phenomenon validates our cyber-physical approach, where a controller that is designed to mitigate imperfections in the communication channels, together with a network manager that is aware of the performance of the physical plant, jointly result in an efficient and safe control system.

Figure 13 is analogous to Figure 9, but it considers the physical disturbance described above. Under sensor disturbance, fixed 2Txs results in one unstable simulation among 40, whereas none of the other algorithms produce unstable simulations. Yet, HC results in executions with MAE comparable to constantly having a \#Tx equal to 3, as shown in Figure 13(a), while achieving an average \#Tx of 2.5, as shown in Figure 13(b). Figure 14 shows the ratio for the \#Actual Tx. HC also obtains the highest ratios of 0 and 1 actual $T x$ per packet, since the system performances sometimes are acceptable even though the PDR is not high enough. Occasionally, HC increases the \#Actual Tx to 3 and 4 in an effort to handle physical attacks. According to Figure 13(c) and 13(d), the \#Actual Tx of actuation WSAN nodes and the system lifetime of the WSAN are almost equal to the baseline of fixed 2 Txs. Compared to PN, HC is significantly more efficient, validating our principle of choosing the network configuration based on the performance of the physical plant.

Figure 15 also presents similar results as Figure 11, in that the data points of $\mathrm{HC}$ concentrate in the bottom left area of Figure 15(a) and the bottom right area of Figure 15(b). Therefore, HC allows a WSAN to be more resilient to both cyber and physical attack by adjusting its network configuration when needed. It also prolongs the system lifetime while keeping MAE within reasonable values.

\subsection{Load Positioning System under Both Cyber and Physical Interferences}

To show the generality of our framework, we also run simulations of a realistic system-load positioning system [19, 37], which positions the load (L) using a motor with a ballscrew transmission. 


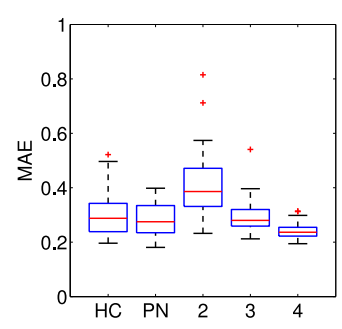

(a) Mean Absolute Error (MAE)

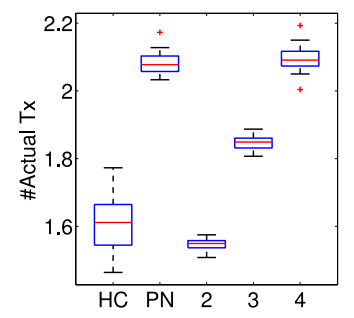

(c) Average number of actual $T x s$ per packet.

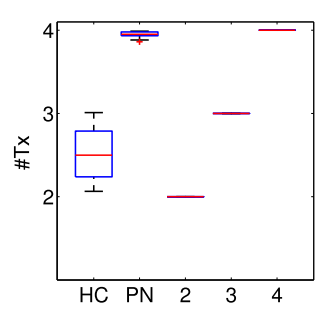

(b) Average number of scheduled Txs.

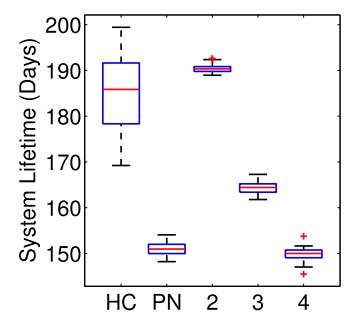

(d) Battery life.

Fig. 13. (a) MAE, (b) average \#Tx, (c) average \#Actual Tx, and (d) battery life for different \#Tx adaptation algorithms under a sensor bias disturbance. The algorithms are our holistic controller (HC), pure network adaptation (PN), and constant transmission numbers equal to 2,3 , and 4, respectively.

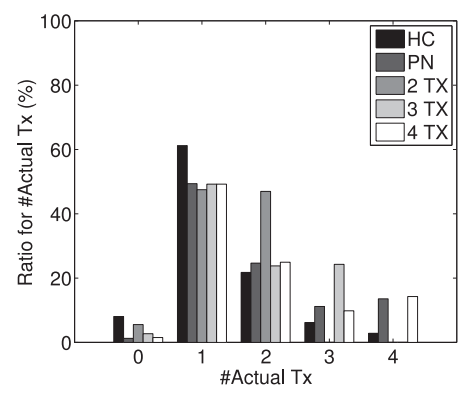

Fig. 14. Ratio for \#Actual Tx under sensor bias disturbance.

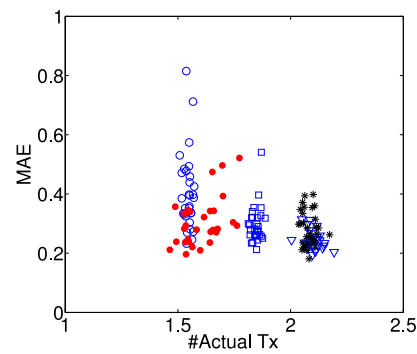

(a) Relationship between MAE and \#Actual Tx.

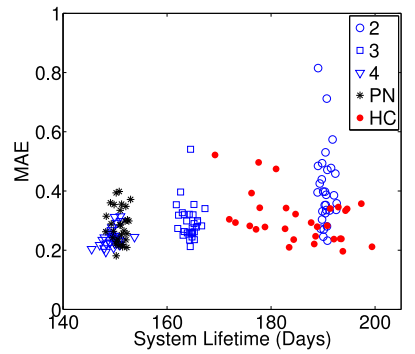

(b) Relationship between MAE and battery life.

Fig. 15. Relationship between MAE and \#Actual Tx as well as system lifetime for different \#Tx adaptation algorithms under sensor bias disturbance. The algorithms are HC (red), PN (black), and fixed \#Tx (blue), respectively. 


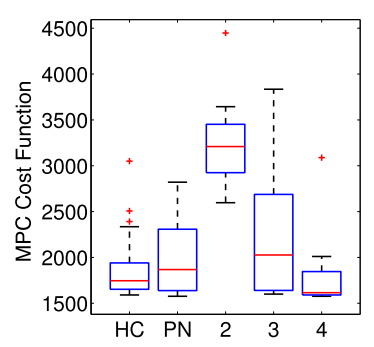

(a) Mean of the cost function.

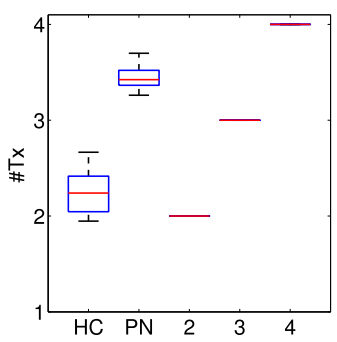

(b) Average number of scheduled Txs.

Fig. 16. (a) Control cost, (b) average \#Tx for different \#Tx adaptation algorithms under sensor bias disturbance. The algorithms are our holistic controller (HC), pure network adaptation (PN), and constant transmission number equal to 2,3 , and 4 , respectively.

The motor is attached rigidly to a movable base platform (B). The load positioning is a four-state nonlinear system as described in Reference [19]. When the system is operated at the low frequencies found in industrial settings, the stiffness of the ballscrew and the potential energy stored in it are neglected in the model. The system can be simplified as a four-state linear system [37]. The state vector is defined as $x_{t}=\left[x_{L}(t) \dot{x_{L}}(t) x_{B}(t) \dot{x_{B}}(t)\right]$, where $x_{L}$ is the displacement of the load relative to the base platform, $x_{B}$ is the absolute displacement of the base platform, and $\dot{x_{L}}$ and $\dot{x_{B}}$ are the speeds of the relative and absolute movements accordingly. We would like to stabilize the states of the load positioning system to the origin in this case study. The dynamics of the system are defined as in Equation (1), where

$$
A=\left[\begin{array}{cccc}
0 & 1 & 0 & 0 \\
0 & -d_{L}\left(\frac{1}{m_{L}}+\frac{1}{m_{B}}\right) & \frac{k_{B}}{m_{B}} & \frac{d_{B}}{m_{B}} \\
0 & 0 & 0 & 1 \\
0 & \frac{d_{L}}{m_{B}} & -\frac{k_{B}}{m_{B}} & -\frac{d_{B}}{m_{B}}
\end{array}\right], \quad B=\left[\begin{array}{c}
0 \\
\frac{1}{m_{L}}+\frac{1}{m_{B}} \\
0 \\
-\frac{1}{m_{B}}
\end{array}\right], \quad \text { and } \quad C=\left[\begin{array}{l}
1 \\
0 \\
0 \\
0
\end{array}\right]^{T} \text {. }
$$

Here, $d_{L}=15, m_{L}=600, d_{B}=10, m_{B}=20$, and $k_{B}=5$ are system parameters of the load and base platform, such as the mass, damping, and stiffness. In this case study, the parameters of the MPC scheme in Equation (2) are chosen as follows:

$$
\begin{aligned}
& Q=\left.\begin{array}{cccc}
0.1 & 0 & 0 & 0 \\
0 & 0.1 & 0 & 0 \\
0 & 0 & 0.1 & 0 \\
0 & 0 & 0 & 0.1
\end{array}\right], \quad S=\left[\begin{array}{cccc}
13.16 & 170.30 & 8.07 & 169.70 \\
170.30 & 4041.1 & 85.98 & 4055.8 \\
8.07 & 85.98 & 7.38 & 86.82 \\
169.70 & 4055.8 & 86.82 & 4072.4
\end{array}\right], \\
& K=\left[\begin{array}{llll}
-1.40 & -26.99 & -0.47 & -26.70]
\end{array}\right.
\end{aligned}
$$

Also, $R=0.05, N=12, \mathcal{X}=\mathbb{R}^{4}$, and $\mathcal{U}=\{|u| \leq 40\}$, where $S, K$, and $\mathcal{X}_{f}$ are computed as described in Lemma 4.1 with $\beta=1.1$. We set the frequencies of the WSAN, the MPC controller, and the KF state observer in our simulations to $4 \mathrm{~Hz}$.

We have run simulations of the load positioning system under both cyber (background noise is $-75 \mathrm{dbm}$ in the first $250 \mathrm{~s}$, and $-78 \mathrm{dbm}$ from $250 \mathrm{~s}$ to $700 \mathrm{~s}$ ) and physical (sensor bias from $370 \mathrm{~s}$ to 420 s) interferences. Again, we conduct five groups of simulations using WCPS as in Sections 6.2 and 6.3. We adopt the cost function of MPC (Figure 16(a)) as a metric of control performance, and we use the average number of scheduled Txs (Figure 16(b)) to measure the cost of the WSN. As concluded in Figure 16(a), HC can achieve comparable control performance to that of constantly having a \#Tx equal to 4, while achieving an average scheduled \#Tx of 2.3, as shown in Figure 16(b). 
(a)
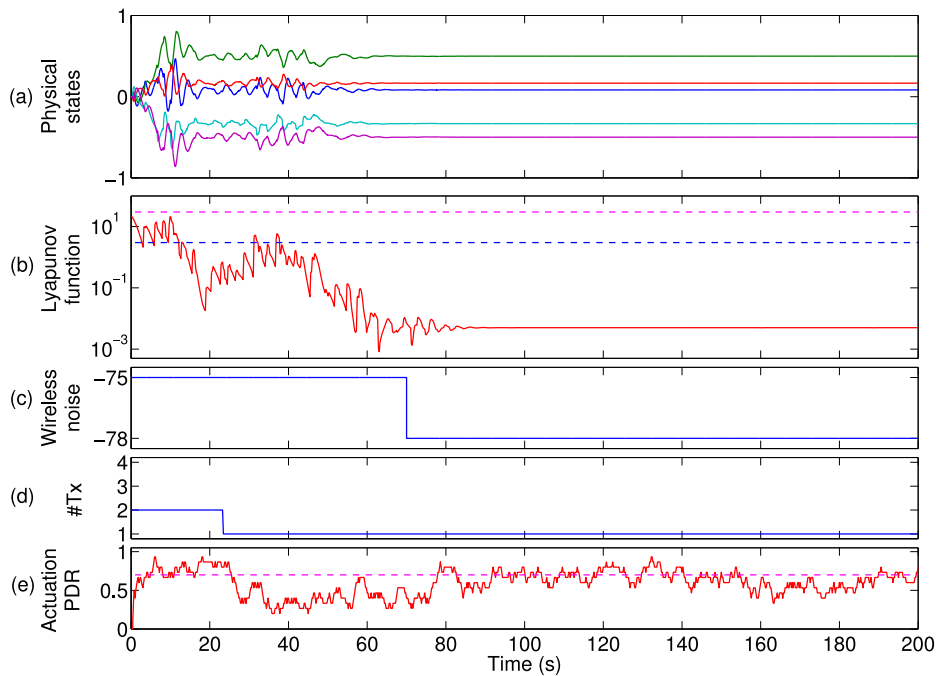

Fig. 17. Open-loop stable plant under wireless disturbance.

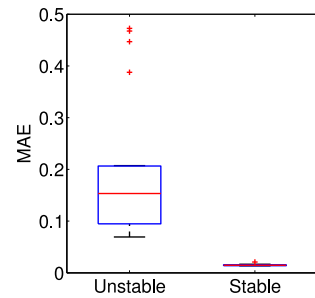

(a) Mean Absolute Error (MAE).

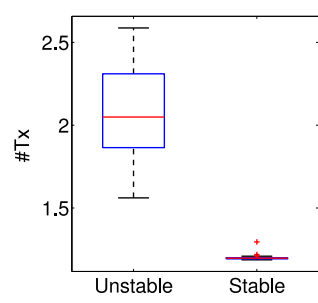

(b) Average scheduled \#Tx per packet.

Fig. 18. (a) MAE. (b) Scheduled \# Tx of open-loop unstable and open-loop stable plants, respectively, under wireless interference. The \#Tx adaptation algorithm is HC.

\subsection{Multi-loop Control System}

In this section, to form a multi-loop simulation, we include an open-loop stable plant that shares the WSAN with the plant described in Section 6.1. For this plant, the set of eigenvalues of $A$ is equal to $\{0.4,0.6,0.96+0.02 i, 0.96-0.02 i, 0.8\}$. Since all eigenvalues are inside the unit circle, the plant is open-loop stable. The control loop of the plant described in Section 6.1 uses the same sensing and actuation flows as in previous sections. The added control loop of the open-loop stable plant uses another pair of sensing and actuation flows in the WSAN. Figure 17 shows the simulation results of the added control loop under the same wireless interference as in Section 6.2.

As is shown in Figure 17(d), during the transient state, the \#Tx is adjusted to 2 for the first $23 \mathrm{~s}$. Since the plant is open-loop stable, the Lyapunov function in Figure 17(b) quickly decreases, and the \#Tx remains at 1 after the transient state ends. It is worth noting that, for open loop stable plants that stabilize at the equilibrium point, the system can retain perfect performance even when the network drops most of its packets. As is shown in Figure 18, the open-loop stable system can achieve smaller MAE with fewer \# Tx, which indicates that our holistic management framework is effective in allocating WSAN resources for the multi-loop system, based on the characteristics of physical plants. In this case, it is possible to allocate those network resources to other lower priority 
applications, such as network health reports. However, the lower priority applications would have to be preempted if the actuation packets claim the network resources. It is also possible to adopt other real-time scheduling and routing methods to balance the allocation of the network resources to multiple control loops based on their properties, such as open-loop stability, time constant, and runtime Lyapunov function. We will address those issues in our future work.

\section{CONCLUSIONS}

In this article, we have proposed a holistic cyber-physical management framework to enhance the dependability of WCSs under both cyber and physical disturbances. The holistic management approach coordinates the physical control and network management mechanisms to safely control the physical plant while efficiently allocating wireless network resources. We then design a concrete holistic controller that considers the worst-case evolution of the Lyapunov function of the plant under ideal network conditions, together with the runtime PDR of the wireless network, and that decides the number of transmissions for each wireless flow. We have implemented the holistic controller and the network management mechanism in the WCPS simulator. A case study that systematically explores both control and wireless performances has been presented using real-world wireless traces. Simulation results show that our holistic controller is capable of maintaining safe physical operation in the presence of sensor disturbances and significant wireless interference. These results shed light on a new family of dependable cyber-physical systems that provides dependable control while efficiently allocating wireless network resources.

\section{REFERENCES}

[1] Saurabh Amin, Alvaro A. Cárdenas, and S. Shankar Sastry. 2009. Safe and secure networked control systems under denial-of-service attacks. In Proceedings of the International Workshop on Hybrid Systems: Computation and Control. Springer, 31-45.

[2] José Araújo, Adolfo Anta, Manuel Mazo Jr., João Faria, Aitor Hernandez, Paulo Tabuada, and Karl Henrik Johansson. 2011. Self-triggered control over wireless sensor and actuator networks. In Proceedings of the 2011 International Conference on Distributed Computing in Sensor Systems and Workshops. IEEE, 1-9. DOI : http://dx.doi.org/10.1109/DCOSS 2011.5982135

[3] Jia Bai, Emeka P. Eyisi, Fan Qiu, Yuan Xue, and Xenofon D. Koutsoukos. 2012. Optimal cross-layer design of sampling rate adaptation and network scheduling for wireless networked control systems. In Proceedings of the International Conference on Cyber-Physical Systems. IEEE, 107-116.

[4] Daniele Bernardini and Alberto Bemporad. 2008. Energy-aware robust model predictive control based on wireless sensor feedback. In Proceedings of the 47th IEEE Conference on Decision and Control. 3342-3347.

[5] Dimitri P. Bertsekas. 2005. Dynamic Programming and Optimal Control, Vol. I (3rd ed.). Athena Scientific.

[6] Md Zakirul Alam Bhuiyan, Guojun Wang, Jiannong Cao, and Jie Wu. 2015. Deploying wireless sensor networks with fault-tolerance for structural health monitoring. IEEE Trans. Comput. 64, 2 (2015), 382-395.

[7] Md Zakirul Alam Bhuiyan, Guojun Wang, Jie Wu, Jiannong Cao, Xuefeng Liu, and Tian Wang. 2017. Dependable structural health monitoring using wireless sensor networks. IEEE Trans. Depend. Secur. Comput. 14, 4 (2017), 363376.

[8] Stephen Boyd and Lieven Vandenberghe. 2004. Convex Optimization. Cambridge University Press.

[9] Panagiotis D. Christofides and Nael H. El-Farra. 2005. Control of Nonlinear and Hybrid Process Systems: Designs for Uncertainty, Constraints and Time-Delays, Vol. 324. Springer, New York.

[10] Cyber-Physical Systems Laboratory, Washington University in St. Louis. 2016. Wireless Cyber Physical Simulator. Retrieved December 27, 2016 from http://wcps.cse.wustl.edu.

[11] Burak Demirel, Arda Aytekin, Daniel E. Quevedo, and Mikael Johansson. 2015. To wait or to drop: On the optimal number of retransmissions in wireless control. In Proceedings of the European Control Conference. IEEE, 962-968.

[12] Burak Demirel, Zhenhua Zou, Pablo Soldati, and Mikael Johansson. 2011. Modular co-design of controllers and transmission schedules in wirelessHART. In Proceedings of the IEEE Conference on Decision and Control and European Control Conference. 5951-5958.

[13] Burak Demirel, Zhenhua Zou, Pablo Soldati, and Mikael Johansson. 2014. Modular design of jointly optimal controllers and forwarding policies for wireless control. IEEE Trans Automat. Control 59, 12 (2014), 3252-3265. 
[14] Mauro Franceschelli, Magnus Egerstedt, and Alessandro Giua. 2008. Motion probes for fault detection and recovery in networked control systems. In Proceedings of the 2008 American Control Conference. IEEE, 4358-4363. DOI : http: //dx.doi.org/10.1109/ACC.2008.4587180

[15] Christoforos N. Hadjicostis and Rouzbeh Touri. 2002. Feedback control utilizing packet dropping network links. In Proceedings of the 41st IEEE Conference on Decision and Control, 2002, Vol. 2. IEEE, 1205-1210.

[16] Song Han, Xiuming Zhu, Aloysius K. Mok, Deji Chen, and Mark Nixon. 2011. Reliable and real-time communication in industrial wireless mesh networks. In Proceedings of 17th Real-Time and Embedded Technology and Applications Symposium. IEEE, 3-12.

[17] HART Communication Foundation. 2017. WirelessHART Specification. Retrieved August 22, 2018 from https:// fieldcommgroup.org/technologies/hart/hart-technology.

[18] G. W. Irwin, J. Chen, A. McKernan, and W. G. Scanlon. 2010. Co-design of predictive controllers for wireless network control. IET Control Theor. Appl. 4, 2 (2010), 186-196.

[19] Yu Jiang, Yebin Wang, Scott A. Bortoff, and Zhong-Ping Jiang. 2015. Optimal codesign of nonlinear control systems based on a modified policy iteration method. IEEE Tran. Neural Netw. Learn. Syst. 26, 2 (2015), 409-414.

[20] Xenofon Koutsoukos, Nicholas Kottenstette, Joe Hall, Panos Antsaklis, and Janos Sztipanovits. 2008. Passivity-based control design for cyber-physical systems. In Proceedings of the International Workshop on Cyber-Physical SystemsChallenges and Applications.

[21] HyungJune Lee, Alberto Cerpa, and Philip Levis. 2007. Improving wireless simulation through noise modeling. In Proceedings of the 6th International Symposium on Information Processing in Sensor Networks. ACM, 21-30.

[22] Bo Li, Yehan Ma, Tyler Westenbroek, Chengjie Wu, Humberto Gonzalez, and Chenyang Lu. 2016. Wireless routing and control: A cyber-physical case study. In Proceedings of the 7th International Conference on Cyber-Physical Systems. ACM/IEEE, 1-10.

[23] Bo Li, Lanshun Nie, Chengjie Wu, Humberto Gonzalez, and Chenyang Lu. 2015. Incorporating emergency alarms in reliable wireless process control. In Proceedings of the 6th International Conference on Cyber-Physical Systems. ACM/IEEE, 218-227.

[24] Bo Li, Zhuoxiong Sun, Kirill Mechitov, Gregory Hackmann, Chenyang Lu, Shirley J. Dyke, Gul Agha, and Billie F. Spencer. April 2013. Realistic case studies of wireless structural control. In Proceedings of the ACM/IEEE International Conference on Cyber-Physical Systems. 179-188.

[25] Merid Lješnjanin, Daniel E. Quevedo, and Dragan Nešić. 2014. Packetized MPC with dynamic scheduling constraints and bounded packet dropouts. Automatica 50, 3 (2014), 784-797.

[26] Chenyang Lu, Abusayeed Saifullah, Bo Li, Mo Sha, Humberto Gonzalez, Dolvara Gunatilaka, Chengjie Wu, Lanshun Nie, and Yixin Chen. 2016. Real-time wireless sensor-actuator networks for industrial cyber-physical systems. Proc. IEEE 104, 5 (2016), 1013-1024.

[27] David Q. Mayne, James B. Rawlings, Christopher V. Rao, and Pierre O. M. Scokaert. 2000. Constrained model predictive control: Stability and optimality. Automatica 36, 6 (2000), 789-814.

[28] Manuel Mazo and Paulo Tabuada. 2008. On event-Triggered and self-triggered control over sensor/actuator networks. In Proceedings of the 47th IEEE Conference on Decision and Control. 435-440. DOI: http://dx.doi.org/10.1109/ CDC.2008.4739414

[29] Luis Antonio Montestruque and Panos Antsaklis. 2004. Stability of model-based networked control systems with time-varying transmission times. IEEE Trans. Automat. Control 49, 9 (2004), 1562-1572.

[30] Sirajum Munir, Shan Lin, Enamul Hoque, S. M. Nirjon, John A. Stankovic, and Kamin Whitehouse. 2010. Addressing burstiness for reliable communication and latency bound generation in wireless sensor networks. In Proceedings of the 9th ACM/IEEE International Conference on Information Processing in Sensor Networks. ACM, 303-314.

[31] Tony O’donovan, James Brown, Felix Büsching, Alberto Cardoso, José Cecílio, Pedro Furtado, Paulo Gil, Anja Jugel, Wolf-Bastian Pöttner, Utz Roedig, and others. 2013. The ginseng system for wireless monitoring and control: Design and deployment experiences. ACM Trans. Sensor Netw. 10, 1 (2013), 4.

[32] Miroslav Pajic, Shreyas Sundaram, Jerome Le Ny, George J. Pappas, and Rahul Mangharam. 2012. Closing the loop: A simple distributed method for control over wireless networks. In Proceedings of the 11th International Conference on Information Processing in Sensor Networks. ACM, 25-36.

[33] Wolf-Bastian Pöttner, Hans Seidel, James Brown, Utz Roedig, and Lars Wolf. 2014. Constructing schedules for timecritical data delivery in wireless sensor networks. ACM Trans. Sensor Netw. 10, 3 (2014), 44.

[34] Abusayeed Saifullah, Chengjie Wu, Paras Babu Tiwari, You Xu, Yong Fu, Chenyang Lu, and Yixin Chen. 2014. Near optimal rate selection for wireless control systems. ACM Trans. Embed. Comput. Syst. 13, 4s (2014), 128.

[35] Luca Schenato, Bruno Sinopoli, Massimo Franceschetti, Kameshwar Poolla, and S. Shankar Sastry. 2007. Foundations of control and estimation over lossy networks. Proc. IEEE 95, 1 (2007), 163-187. DOI : http://dx.doi.org/10.1109/JPROC. 2006.887306 
[36] Pierre O. M. Scokaert and James B. Rawlings. 1998. Constrained linear quadratic regulation. IEEE Trans. Automat. Control 43, 8 (1998), 1163-1169.

[37] Vijay Shilpiekandula, Scott A. Bortoff, John C. Barnwell, and Khalid El Rifai. 2012. Load positioning in the presence of base vibrations. In Proceedings of the American Control Conference (ACC'12). IEEE, 6282-6287.

[38] Bruno Sinopoli, Luca Schenato, Massimo Franceschetti, Kameshwar Poolla, Michael I. Jordan, and S. Shankar Sastry. 2004. Kalman filtering with intermittent observations. IEEE Trans. Automat. Control 49, 9 (2004), 1453-1464. DOI: http://dx.doi.org/10.1109/TAC.2004.834121

[39] IEC Std. 2016. Industrial communication network - Wireless communication network and communication profiles WirelessHART. Int. Electrotech. Comm. 2.0 IEC 62591.

[40] Paulo Tabuada. 2007. Event-triggered real-time scheduling of stabilizing control tasks. IEEE Trans. Automat. Control 52, 9 (2007), 1680-1685.

[41] The Mathworks Inc. 2017. Simulink. Retrieved May 21, 2017 from http://www.mathworks.com/products/simulink.

[42] Alphan Ulusoy, Ozgur Gurbuz, and Ahmet Onat. 2011. Wireless model-based predictive networked control system over cooperative wireless network. IEEE Trans. Ind. Inform. 7, 1 (2011), 41-51.

[43] ISA 100 WIRELESS. 2017. Wireless Systems for Automation. Retrieved May 25, 2017 from http://www.isa100wci.org.

[44] Emmanuel Witrant, Pan Gun Park, Mikael Johansson, Carlo Fischione, Karl Henrik Johansson, et al. 2007. Predictive control over wireless multi-hop networks. In Proceedings of the IEEE International Conference on Control Applications. 1037-1042.

[45] Chengjie Wu, Dolvara Gunatilaka, Abusayeed Saifullah, Mo Sha, Paras Babu Tiwari, Chenyang Lu, and Yixin Chen. 2016. Maximizing network lifetime of wirelessHART networks under graph routing. In Proceedings of the International Conference on Internet-of-Things Design and Implementation. IEEE, 176-186.

[46] Chengjie Wu, Dolvara Gunatilaka, Mo Sha, Chenyang Lu, and Yixin Chen. 2015. Conflict-aware real-time routing for industrial wireless sensor-actuator networks. In Technical Report WUCSE-2015-005. All Computer Science and Engineering Research. http://openscholarship.wustl.edu/cse_research/507.

[47] Zigbee Alliance. 2017. ZigBee Alliance. Retrieved April 9, 2017 from http://www.zigbee.org.

Received April 2017; revised November 2017; accepted January 2018 